\title{
Deporte activo y pasivo: ¿Una relación de conveniencia?*
}

\section{FERNANDO LERA-LÓPEZ}

Departamento de Economía, UNIVERSIDAD PÚBLICA DE NAVARRA, ESPAÑA. E-mail: lera@unavarra.es

\section{MARÍA JOSÉ SUÁREZ}

Departamento de Economía, UNIVERSIDAD DE OVIEDO, ESPAÑA. E-mail: msuarezf@uniovi.es

\section{RESUMEN}

Este es un trabajo de carácter empírico que estudia la relación entre las decisiones individuales relativas al deporte activo y pasivo, a partir de una encuesta realizada en la Comunidad Foral de Navarra en el año 2007. En primer lugar, se analiza la práctica deportiva y la asistencia a espectáculos deportivos mediante un modelo Probit bivariante y un Logit multinomial . En segundo lugar, se estudia la relación entre los gastos asociados a dichas actividades mediante un modelo Tobit bivariante. Los resultados muestran una correlación positiva y significativa entre deporte activo y pasivo, una vez controladas las variables socio-demográficas.

Palabras clave: Participación deportiva, asistencia deportiva, gasto deportivo.

\section{Active and Passive Sport: Is it a Marriage of Convenience?}

\begin{abstract}
This empirical paper studies the relationship between individual decisions regarding active and passive sport activities, using a survey conducted in the Spanish region of Navarra in 2007. Firstly, we estimate a bivariate Probit model and a multinomial Logit to analyze sports practice and sporting event attendance. Secondly, we apply a bivariate Tobit model to estimate the determinants of individual expenditures related to both activities. In both participation and expenditures, the results show a significant and positive correlation between active and passive sport activities after controlling for socio-demographic variables.
\end{abstract}

Keywords: Sports Practice, Sporting Event Attendance, Expenditure on Sport Activities.

Clasificación JEL: D12, L83

\footnotetext{
* Fernando Lera-López agradece el apoyo financiero del Consejo Superior de Deportes en el desarrollo del Proyecto 32/UPB20/06.
}

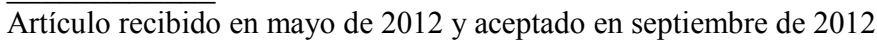

Artículo disponible en versión electrónica en la página www.revista-eea.net, ref. ə-30217 


\section{INTRODUCCIÓN}

En los últimos años, ha crecido de manera significativa la importancia económica del deporte en España (Benítez Rochel y Lacomba Arias, 2012; Bosch et al., 2012). En el año 1990 las primeras estimaciones indicaban que el peso del deporte en el PIB era del 1,2\% (Alonso et al., 1991), mientras que en el 2006 la cifra se eleva a 2,38\% (Lera-López et al., 2008).

Esta importancia económica del deporte está íntimamente relacionada con el significativo crecimiento de la participación deportiva en muchos países europeos, en un contexto de una activa política deportiva y una fuerte inversión en infraestructura deportiva. Asimismo, los efectos positivos sobre la salud y el bienestar asociados a la realización de actividad física han contribuido a que una población cada vez más sedentaria se animara a su práctica. En el caso español, la participación deportiva se ha incrementado desde el $34 \%$ en el año 1985 al 45\% en el año 2010 (García y Llopis, 2011). La asistencia a eventos deportivos no ha permanecido ajena a esta tendencia creciente. Por ejemplo, la asistencia a los campos de fútbol en España se ha incrementado un 7\% entre la temporada 1999-2000 y la temporada 2006-2007 (European Football Statistics, 2009).

En este contexto, se han ido desarrollando distintos trabajos empíricos con el objetivo de determinar los patrones socio-económicos asociados a la práctica deportiva y al gasto destinado a la misma. El análisis de los determinantes de las decisiones individuales relacionadas con el deporte es importante para las autoridades públicas. Por un lado, el deporte tiene efectos positivos sobre el estado de salud y, por tanto, la práctica generalizada de actividad física puede reducir el gasto público en materia de sanidad. Por otro lado, los espectáculos deportivos constituyen una actividad que puede contribuir a la integración social y al desarrollo económico.

En nuestro país, los trabajos empíricos sobre las decisiones individuales de practicar ejercicio físico y de asistir a eventos deportivos no son numerosos, y aún son más escasas las aportaciones sobre el tema del gasto individual en actividades deportivas debido, en este último caso, a la falta de bases de datos apropiadas.

Cabría esperar que los individuos que tienen gustos favorables a la práctica deportiva, también tiendan a acudir con mayor probabilidad a espectáculos deportivos y viceversa Nuestro trabajo tiene un doble objetivo. En primer lugar, pretendemos contrastar si existe relación entre la práctica y la asistencia a eventos deportivos, a través del análisis de la correlación entre ambas decisiones, una vez que se ha tenido en cuenta el efecto de las variables de tipo sociodemográfico. Para ello se estima un Probit bivariante de asistencia y práctica de deporte. Además, para analizar los determinantes conjuntos de la asistencia a eventos deportivos y la realización de actividades deportivas también hemos planteado un modelo Logit multinomial. En segundo lugar, analizamos la co- 
rrelación entre el gasto en asistencia a espectáculos deportivos y el gasto asociado a la práctica deportiva mediante un Tobit bivariante, que tiene en cuenta que hay una proporción significativa de individuos que no realizan gastos en estas actividades.

La característica diferencial de nuestro artículo en relación a la literatura económica existente para nuestro país es el análisis conjunto del deporte activo y pasivo en sus dos facetas: participación y gasto. La base de datos empleada es una Encuesta de Participación y Gasto Deportivo realizada en Navarra en el año 2007 a un total de 831 personas adultas. A pesar de tratarse de una encuesta regional y con un tamaño muestral relativamente reducido, tiene la ventaja de que ofrece amplia información sobre participación individual y gasto en actividades relacionadas con la práctica deportiva y con la asistencia a eventos deportivos, entre otros temas.

El contenido del artículo se organiza del siguiente modo. En primer lugar, se hace una revisión de las aportaciones empíricas sobre el tema. Seguidamente se especifican los modelos econométricos a estimar. En la sección 4 se ofrece un análisis descriptivo y se presentan los resultados empíricos obtenidos. Por último, se exponen las principales conclusiones del trabajo.

\section{REVISIÓN DE LA LITERATURA}

Las actividades deportivas individuales pueden tener un carácter activo o pasivo. El deporte activo implica la práctica de actividades deportivas, mientras que el deporte pasivo hace referencia fundamentalmente a la asistencia a espectáculos deportivos como espectador. La demanda individual de actividades deportivas activas o pasivas se puede analizar desde dos vertientes: la práctica (si participa o no, con qué frecuencia o cuánto tiempo se destina a ella) y el gasto asociado a la misma.

\subsection{Determinantes de la práctica deportiva activa y pasiva}

A nivel internacional se han publicado numerosas investigaciones que estudian los factores explicativos de las decisiones individuales de practicar deporte, recogidas en distintos trabajos recopilatorios (Downward et al., 2009, 2012; Breuer et al., 2010). El análisis de la asistencia a espectáculos deportivos empieza más tarde y se ha concentrado tradicionalmente en analizar determinados deportes profesionales, como el fútbol, el béisbol, el baloncesto y rugby americano (García y Rodríguez, 2002, 2009; Byon et al., 2010).

En el caso de España, se han publicado trabajos relativos a la liga española de fútbol (García y Rodríguez, 2002), a la asignación individual de tiempo a la práctica deportiva (Lera-López y Rapún, 2007; García et al., 2011), a activida- 
des deportivas de carácter activo y pasivo (Muñiz et al., 2011), así como la pertenencia a entidades deportivas (Sigüenza y Mariel, 2012).

La evidencia empírica muestra como la participación deportiva decrece con la edad (Breuer y Wicker, 2008; Downward et al., 2011, 2012) debido a limitaciones físicas y cambios en las preferencias de ocio de la gente mayor (Barber y Havitz, 2001). Sin embargo, la edad parece estar relacionada positivamente con la asistencia a eventos deportivos (Zhang et al., 2003), aunque con diferencias entre deportes profesionales y amateur (Thrane, 2001).

Por otro lado, los hombres practican más deporte que las mujeres (Lera-López y Rapún, 2007; Breuer y Wicker, 2008; Eberth y Smith, 2010; Downward et al., 2012; Kokolakakis et al., 2012) y asisten más a espectáculos deportivos (Fernández y Prieto, 2001; Thrane, 2001; Montgomery y Robinson, 2010). Estas diferencias tradicionalmente se atribuyen a factores biológicos, culturales y sociales, reflejando diferencias en la asunción de responsabilidades en el hogar y el trabajo. No obstante, algunos trabajos señalan diferencias según tipología de deportes y países (Fridberg, 2010; Humphreys y Ruseski, 2010; Van Tuyckom et al., 2010; Scheerder y Vos, 2011).

La educación es otra variable tradicionalmente incluida en estos trabajos, mostrando una relación positiva con la asistencia a espectáculos deportivos (White y Wilson, 1999; Wilson, 2002; Zhang et al., 2003; Montgomery y Robinson, 2010) y con la práctica deportiva (Breuer y Wicker, 2008; Eberth y Smith, 2010; Fridberg, 2010; Downward et al., 2011; 2012; García et al., 2011; Scheerder y Vos, 2011 y Kokolakakis et al., 2012). Un alto nivel educativo puede llevar a una mayor conciencia de los efectos beneficiosos de la práctica deportiva en la salud, por ejemplo, a la vez que está asociado a mayores ingresos y recursos económicos, que pueden favorecer una mayor práctica. Asimismo, mayores niveles educativos suelen implicar ocupaciones laborales más sedentarias (Fridberg, 2010).

Otra variable relevante es la disponibilidad de tiempo. Dado que el tiempo es finito, cualquier incremento del tiempo dedicado al deporte supone una disminución del tiempo destinado a otras actividades de ocio, al trabajo y a la familia. La influencia de este factor suele analizarse de manera indirecta mediante el nivel de ingresos, la ocupación y la estructura familiar. Distintos trabajos han puesto de manifiesto como estar ocupado está negativamente relacionado con la participación deportiva (Downward, 2007; Breuer y Wicker, 2008; Hovemann y Wicker, 2009 y Eberth y Smith, 2010) mientras que la evidencia empírica relativa a la asistencia a espectáculos deportivos es dispersa (Serrano, 1999; Fernández y Prieto, 2001 y Zhang et al., 2003). Esa misma falta de consenso se manifiesta al analizar el nivel de ingresos y la asistencia a eventos deportivos. La evidencia empírica recogida por García y Rodríguez (2009); Downward et al. (2009) y Montgomery y Robinson (2010) muestra que muchos deportes pro- 
fesionales pueden ser considerados como bienes normales (por tanto, la elasticidad-renta es positiva, aunque generalmente inferior a la unidad), aunque con excepciones (Falter y Pérignon, 2000) y con menor evidencia en el deporte amateur (White y Wilson, 1999).

Asimismo, la estructura familiar, medida a través del estado civil y el tamaño del hogar se ha convertido en un factor determinante de la práctica deportiva. Las personas casadas tienden a practicar menos deporte (García et al., 2011), aunque con diferencias según género (Eberth y Smith, 2010). El tamaño del hogar también suele estar negativamente relacionado con la asistencia a eventos deportivos (Zhang et al., 2003) y con la práctica deportiva (Downward, 2007; Humphreys y Ruseski, 2007), si bien hay trabajos recientes que muestran una relación positiva (Lechner, 2009; Downward y Rasciute, 2010). Asimismo, la presencia de niños en el hogar tiende a disminuir la probabilidad de práctica deportiva (García et al., 2011) y a cambiar la tipología de los deportes practicados (Humphreys y Ruseski, 2010).

Finalmente, distintos trabajos analizan la influencia positiva de la disponibilidad de instalaciones en la demanda deportiva (Wicker et al., 2009; Downward y Rasciute, 2010). Otros trabajos incluyen el tamaño de la población como variable proxy de la oferta deportiva. No obstante, su influencia no está clara. Por un lado, en las zonas urbanas la disponibilidad de instalaciones deportivas es mayor y más variada, incentivando su uso (Hovemann y Wicker, 2009 y García et al., 2011), pero por otro lado, también hay mayor variedad de alternativas de ocio que compiten con la práctica deportiva, pudiendo tener un efecto negativo global (Scheerder et al., 2005). En el caso de la asistencia a eventos deportivos el efecto positivo de la oferta es superior que la mayor diversidad de alternativas de ocio, por lo que el efecto global es positivo (Falter y Pérignon, 2000; Winfree et al., 2004).

\subsection{Determinantes del gasto en deporte activo y pasivo}

La literatura que analiza el gasto individual en bienes y servicios relacionados con la práctica deportiva es mucho más limitada, probablemente por la escasez de bases de datos que dispongan de dicha información (Downward et al., 2009; Breuer et al., 2010; Scheerder et al., 2011). La evidencia específica sobre el gasto en asistencia a eventos deportivos es aún más escasa, centrada mayoritariamente en trabajos de carácter descriptivo. Entre los últimos trabajos con análisis econométricos podemos citar a Loyland y Ringstad (2009) y Pawlowski y Breuer (2011).

En el caso de España, en el ámbito del gasto individual en deporte cabe mencionar a Lera-López y Rapún $(2005,2007)$, que analizan los gastos en deporte activo y Lera-López et al. (2011) que se ocupan del gasto en asistencia a eventos deportivos. 
Lógicamente, distintos trabajos han mostrado una relación positiva entre la frecuencia y la intensidad de la práctica deportiva y los gastos asociados a la misma (Taks et al., 1999; Davies, 2002; Lera-López y Rapún, 2007; Wicker et al., 2010; Scheerder et al., 2011). Por ejemplo, Wicker et al. (2010) estiman que un incremento de una hora diaria en la práctica deportiva implica un aumento del gasto en deporte de 263 euros al año.

El gasto en deporte activo parece estar asociado negativamente con la edad, dada la menor participación deportiva de las personas mayores (Breuer et al., 2010), de la misma manera que los hombres tienden a gastar más en deporte que las mujeres (Lamb et al., 1992; Taks et al., 1995; Lera-López y Rapún, 2005, 2007; Scheerder et al., 2011). La evidencia empírica es menos consistente al analizar la influencia de la educación. Mientras que algunos autores señalan que el gasto en deporte disminuye con el nivel educativo (Taks et al., 1999), otros presentan evidencia de una relación positiva (Lera-López y Rapún, 2005; Scheerder et al., 2011).

Generalmente se obtiene una relación directa entre el nivel de ingresos y el gasto en deporte activo (Lamb et al., 1992; Taks et al., 1995, 1999; Lera-López y Rapún, 2005, 2007). Loyland y Ringstad (2009), que analizan conjuntamente el gasto en deporte activo y pasivo en Noruega, muestran resultados semejantes. Asimismo, señalan diferencias según el tamaño del hogar y el tamaño de la población, con una relación negativa entre estas variables y el gasto total en deporte activo y pasivo.

La evidencia empírica centrada en los determinantes de la asistencia a eventos deportivos en general, es aún más limitada. Cannon y Ford (2002) encuentran, para Estados Unidos, que el nivel económico y la presencia de menores en el hogar influyen positivamente en el gasto, mientras que otras variables, como la educación o el estado civil no resultan significativas.

Trabajos posteriores como los de Pawlowski y Breuer (2011) y Lera-López et al. (2011) han profundizado en estas relaciones. Pawlowski y Breuer (2011) obtienen una relación negativa entre el porcentaje de gasto en eventos deportivos en Alemania y el tamaño del municipio, el nivel educativo de las personas y la presencia de niños y jóvenes en el hogar. Variables como la edad, la ocupación o el estado civil no son significativas. Un trabajo posterior de los mismos autores (Pawlowski y Breuer, 2012) estima unas elasticidades del gasto en eventos deportivos superiores a la unidad. Para España, Lera-López et al. (2011) concluyen que el gasto en asistencia a eventos deportivos de carácter profesional y amateur es mayor para los hombres, y también aumenta con el nivel de educación y con la renta. Sin embargo, variables que recogen la disponibilidad de tiempo y que eran significativas para explicar la asistencia a eventos deportivos no lo son para explicar el gasto asociado a los mismos (ocupación, estado civil y número de menores en el hogar). 


\subsection{Relaciones entre deporte activo y pasivo}

La evidencia empírica nos sugiere que el deporte activo y pasivo pueden estar determinados por la interacción de factores económicos (nivel de ingresos, ocupación) así como por características individuales y sociales (género, edad, nivel educativo, etc.), entre otras variables. A pesar de las similitudes entre los posibles determinantes de ambas actividades, en la literatura económica el deporte pasivo y activo se suelen analizar de forma independiente (Taks y Scheerder, 2006), prestándose escasa atención a la relación entre participación deportiva y asistencia a eventos deportivos (Zhang et al., 1997; Thrane, 2001).

Desde un punto de vista teórico y descriptivo, existen dos enfoques al plantear una posible relación entre deporte activo y pasivo. Por un lado, se argumenta que ambas prácticas presentan similitudes en términos de motivaciones, actitudes y beneficios asociados a su práctica (Zilmann et al., 1979 y Sloan, 1989). Bajo esta argumentación ambas prácticas estarían positivamente relacionadas y entre las dos se retroalimentarían. Así, Sloan (1989) señala que la asistencia a espectáculos deportivos debe incrementar la posibilidad de practicar deporte. Thrane (2001), usando datos de los países escandinavos, muestra que la tendencia es recíproca, y que la participación deportiva también incrementa la posibilidad de asistencia a espectáculos deportivos.

Sin embargo, una segunda corriente señala que ambas formas de disfrutar del tiempo libre no están relacionadas entre sí (Stone, 1971; Shamir y Ruskin, 1984 y Burnett et al., 1993). Este último autor, usando la técnica del Chi-cuadrado, concluye que el deporte activo y el pasivo son independientes uno de otro y que difieren en cuanto a sus determinantes, tanto en términos de motivación y de variables sociales y demográficas, como la edad, el género, la educación o la ocupación, coincidiendo con los análisis realizados por Shamir y Ruskin (1984). Posteriormente, Lines (2007), en un estudio entre jóvenes ingleses, señala una escasa relación entre ambas prácticas.

En algunos trabajos recientes se contrasta la posible relación entre deporte activo y pasivo mediante modelos econométricos que incluyen la asistencia a espectáculos como un factor explicativo de la práctica deportiva o viceversa (Dawson y Downward, 2011; Downward et al., 2011 y 2012). No obstante, este enfoque puede presentar problemas de endogeneidad si existen factores inobservados que afectan simultáneamente a las decisiones de practicar deporte y asistir a espectáculos.

La aportación fundamental de este artículo es el estudio conjunto de la participación deportiva y de la asistencia a espectáculos deportivos, por un lado, y del gasto destinado a deporte activo y pasivo, por otro lado. 


\section{METODOLOGÍA}

En esta sección se especifican, en primer lugar, los modelos econométricos que se emplearán para analizar las decisiones de participación en deporte activo y pasivo y, en segundo lugar, el relativo a los gastos individuales en ambos tipos de actividades deportivas. La estimación de estos modelos permitirá contrastar la hipótesis de partida: la existencia de correlación entre las decisiones individuales de deporte activo y pasivo, así como profundizar en el análisis de los determinantes de la participación y el gasto en actividades deportivas.

\subsection{Decisiones individuales de participación en actividades deportivas activas y pasivas}

En este trabajo se considera que una persona practica deporte cuando realiza, al menos una vez al mes, cualquier tipo de deporte o actividad física, excluyendo caminar. Respecto a la asistencia, se supone que un individuo es participante si ha acudido a eventos deportivos de carácter profesional o amateur en el último año con relativa frecuencia (una vez al mes como mínimo).

En el análisis de las decisiones de participación, planteamos dos especificaciones alternativas, que nos permitirán comprobar la robustez de los resultados. En ambos casos se parte del supuesto de que el objetivo del individuo es maximizar su utilidad.

En la primera especificación, definimos para cada individuo $i$ un índice, $d a_{i}{ }^{*}$, que es igual a la diferencia entre el nivel de bienestar cuando practica deporte y cuando no lo practica. Si el índice es positivo, el individuo participa y, si es negativo, no. De manera análoga, se define un índice para la decisión de asistir a eventos deportivos $\left(d p_{i}{ }^{*}\right)$.

Se supone que la utilidad es función de características personales observadas que pueden influir en los gustos y tiene también un componente aleatorio que recoge la influencia de factores inobservables. En consecuencia, la participación en actividades deportivas activas y pasivas se especifica del siguiente modo:

$$
\begin{aligned}
& d a_{i}^{*}=\alpha X_{i}+\varepsilon_{i}^{a} \\
& d p_{i}^{*}=\beta X_{i}+\varepsilon_{i}^{p}
\end{aligned}
$$

donde $\alpha$ y $\beta$ son vectores de coeficientes a estimar, $X_{i}$ es un vector de variables independientes y $\varepsilon^{a}{ }_{i}$ y $\varepsilon^{p}{ }_{i}$ son términos aleatorios que siguen una distribución normal bivariante, con medias cero, varianzas unitarias y un coeficiente de correlación $\rho$.

Las variables dependientes observadas, $d a_{i}$ y $d p_{i}$, toman el valor uno si el índice correspondiente es positivo, y cero en otro caso: 


$$
\begin{aligned}
& d a_{i}=\left\{\begin{array}{lll}
1 & \text { si } & d a_{i}^{*}>0 \\
0 & \text { en otro caso }
\end{array}\right. \\
& d p_{i}=\left\{\begin{array}{lll}
1 & \text { si } & d p_{i}^{*}>0 \\
0 & \text { en } & \text { otro caso }
\end{array}\right.
\end{aligned}
$$

Dado que tenemos un sistema de dos ecuaciones con variables dependientes binarias, que pueden estar correlacionadas a través de los términos de error, el método de estimación aplicado en este caso es un modelo Probit bivariante ${ }^{1}$.

La segunda especificación parte de la consideración de cuatro alternativas para los individuos:

- no practica deporte ni asiste a espectáculos deportivos de forma regular;

- asiste a eventos deportivos, pero no practica deporte;

- realiza actividades deportivas, pero no acude a espectáculos deportivos;

- practica deporte y asiste a eventos de carácter deportivo de forma regular.

El individuo elige la opción que más utilidad le proporciona de las cuatro, donde la utilidad de cada alternativa depende de características personales y tiene un componente aleatorio. Dado que las alternativas no son ordenadas, hemos aplicado un Logit multinomial, lo que implica asumir que los errores de cada alternativa son independientes y están idénticamente distribuidos siguiendo una distribución de valor extremo tipo $I$. En consecuencia, la probabilidad de que una persona opte por la alternativa $j$ vendría dada por la siguiente expresión:

$$
\operatorname{Pr}(\text { alternativa } \quad j)=\frac{e^{\varphi_{j} X_{i}}}{\sum_{k=1}^{4} e^{\varphi_{k} X_{i}}}, \quad j=1,2,3,4
$$

En la ecuación anterior $\varphi_{k}$ es el vector de parámetros de la alternativa $k^{2}$.

La estimación del Logit multinomial nos ofrece información sobre las variables que determinan la probabilidad de que los individuos practiquen deporte y asistan a eventos deportivos de forma regular y simultánea.

Las variables independientes consideradas en ambos modelos son las usuales en la literatura económica sobre el tema. Se incluyen la edad, el nivel educativo (mediante dos variables binarias de estudios medios y superiores respectivamente), variables dicotómicas de género, estado civil y situación laboral (que son igual a uno para hombres, casados y ocupados, respectivamente), una varia-

\footnotetext{
${ }^{1}$ Para más información, véase Cameron y Trivedi (2009).

${ }^{2}$ Véase Cramer(1991) para más detalles acerca de los modelos Logit.
} 
ble dummy que es igual a uno si el encuestado es estudiante, el número de hijos $\mathrm{y}$ de adultos en el hogar, el estado de salud (igual a uno cuando la persona declara que su estado de salud es bueno o muy bueno), una variable geográfica (igual a uno para residentes en la capital de la región, Pamplona, y comarca) y una variable binaria para fumadores, porque este colectivo puede tener distintas preferencias hacia el deporte, en especial en la práctica de actividades deportivas. En el Apéndice se incluye la definición de todas las variables utilizadas, así como los estadísticos descriptivos de la muestra.

Cabe mencionar que, aunque la base de datos incorpora información sobre la renta familiar neta en intervalos, el porcentaje de falta de respuesta es muy elevado, por lo que su inclusión como variable explicativa reduciría significativamente el tamaño muestral. En consecuencia, se ha incluido la situación laboral como una proxy de la renta, aunque a la hora de interpretar los resultados hay que tener en cuenta que el efecto de otras variables como la educación y el número de adultos puede ser debido, en parte, a su influencia sobre la renta familiar.

\subsection{Gastos individuales en práctica deportiva y en asistencia a eventos deportivos}

La base de datos empleada ofrece información detallada sobre hábitos deportivos y sobre gastos relacionados con ellos. En el ámbito de los gastos, se pregunta a la persona encuestada sobre la cantidad media de dinero destinada a determinados productos o servicios, y se mide el gasto en intervalos.

Partiendo de la información de la encuesta, el gasto en deporte activo, $g a_{i}$, se define como el gasto mensual medio en cuotas o abonos de asociaciones deportivas, en utilización de instalaciones deportivas, en clases o cursos deportivos, en calzado y ropa deportiva orientados a la práctica de ejercicio físico, en material duradero para la práctica deportiva y en desplazamientos, alimentos, bebidas, etc. asociados a la práctica deportiva.

Por otra parte, el gasto en deporte pasivo, $g p_{i}$, incluye el gasto medio mensual en asistencia a eventos deportivos (incluyendo cuotas como socio), y en desplazamientos, refrescos, comidas y productos de promoción adquiridos al acudir a eventos deportivos.

Dado que la encuesta mide los gastos en intervalos, para calcular las variables anteriormente definidas, se calcula el punto medio del intervalo para cada tipo de gasto y se suman los valores obtenidos. Como resultado, se obtienen variables continuas y censuradas, puesto que hay una proporción relevante de ceros.

En la especificación empírica, se parte de dos variables dependientes latentes para el gasto activo y pasivo $\left(g a^{*}\right.$ y $\left.g p^{*}\right)$ que pueden tomar cualquier valor. 
Estas variables son función lineal de un vector de factores explicativos $\left(X_{i}\right)$ y tienen un componente aleatorio $\left(v_{i}^{a}\right.$ y $v_{i}^{p}$, respectivamente):

$$
\begin{aligned}
& g a_{i}^{*}=\delta X_{i}+v_{i}^{a} \\
& g p_{i}^{*}=\lambda X_{i}+v_{i}^{p}
\end{aligned}
$$

En las ecuaciones anteriores $\delta$ y $\lambda$ son vectores de parámetros.

Las variables de gasto observadas, $g a_{i}$ y $g p_{i}$, están censuradas en cero y se definen del siguiente modo:

$$
\begin{gathered}
g a_{i}=\left\{\begin{array}{l}
g a_{i}^{*} \text { si } g a_{i}^{*}>0 \\
0 \quad \text { si } g a_{i}^{*} \leq 0
\end{array}\right. \\
g p_{i}= \begin{cases}g p_{i}^{*} & \text { si } g p_{i}^{*}>0 \\
0 & \text { si } g p_{i}^{*} \leq 0\end{cases}
\end{gathered}
$$

Suponiendo que $v^{a}{ }_{i} \mathrm{y} v^{p}{ }_{i}$ siguen una distribución normal bivariante con coeficiente de correlación $\rho$ y medias nulas, las ecuaciones anteriores se pueden estimar mediante un Tobit bivariante.

Las variables explicativas incluidas en las ecuaciones de gasto coinciden con las de los modelos especificados para analizar la participación que se han expuesto en la sección anterior.

\section{ANÁLISIS EMPÍRICO}

Los modelos econométricos especificados en el apartado anterior se estiman empleando una encuesta regional realizada en Navarra en el año 2007. La encuesta fue llevada a cabo por CIES y financiada por el Consejo Superior de Deportes. En ella se recoge información de 831 personas adultas sobre hábitos y prácticas deportivas activas, participación y gastos en deporte pasivo, valoraciones sobre la deportividad y la política deportiva navarra y, por último, variables de tipo socio-demográfico.

Las muestras seleccionadas para cada uno de los dos modelos econométricos se restringen a aquellas personas para las que se dispone de datos sobre todas las variables consideradas. El tamaño muestral del Probit bivariante y del Logit multinomial es de 810 observaciones y el del Tobit bivariante, de 704 .

En la primera sección de este apartado nos centramos en las decisiones de práctica de deporte y asistencia a espectáculos, mientras que en la segunda sección nos ocupamos de los gastos destinados a cada una de esas actividades. 


\subsection{Análisis empírico de la participación deportiva}

En primer lugar, es interesante analizar cómo se distribuye la muestra en función de su participación en actividades deportivas de carácter activo y pasivo. La Tabla 1 recoge esta información y, de los datos en ella mostrados, se deduce que aproximadamente un $35 \%$ de la población adulta navarra practica deporte o ejercicio físico periódicamente y un $27 \%$ ha asistido a eventos deportivos al menos una vez al mes en el año anterior. La tabla no revela una correlación muy clara entre deporte activo y pasivo. Analizando los datos de la tabla en diagonal, se observa que hay un $64 \%$ de la muestra que, o bien no participa en ninguna de esas actividades o bien participa en ambas, frente al $36 \%$ que realiza una de las dos.

Tabla 1

Distribución de la muestra en función de su participación regular en actividades deportivas activas y pasivas

\begin{tabular}{|l|c|c|c|}
\hline & $\begin{array}{c}\text { No asiste a eventos } \\
\text { deportivos }\end{array}$ & $\begin{array}{c}\text { Asiste a eventos } \\
\text { deportivos }\end{array}$ & $\mathbf{N}^{\circ}$ observaciones \\
\hline No practica deporte & 414 & 114 & 528 \\
\hline Practica deporte & 181 & 101 & 282 \\
\hline N $^{\circ}$ observaciones & 595 & 215 & 810 \\
\hline
\end{tabular}

Fuente: Elaboracion propia.

El Probit bivariante y el Logit multinomial especificados en el apartado anterior nos permiten conocer los determinantes de la probabilidad de practicar deporte y de asistir a eventos deportivos. Asimismo, el Probit bivariante ofrece información sobre la posible correlación entre ambas decisiones.

En la Tabla 2 se presentan los coeficientes y los estadísticos $t$ de Student del modelo Probit bivariante. En general, los resultados son similares a los obtenidos en la literatura sobre el tema.

Los hombres tienen mayor probabilidad que las mujeres de practicar deporte y de asistir a eventos deportivos de forma regular. La probabilidad de realizar deporte es mayor entre los más jóvenes y los estudiantes. Sin embargo, la asistencia habitual a espectáculos deportivos es más frecuente en las personas de edad media (entre 40 y 59 años). La práctica deportiva también aumenta con el nivel de estudios, lo que puede deberse a que las personas más formadas son más conscientes de los beneficios del deporte para la salud y el bienestar. En cambio, la formación no es relevante en la probabilidad de asistir a espectáculos deportivos.

Por otra parte, es más probable que los individuos que declaran un estado de salud bueno o muy bueno practiquen deporte, mientras que los fumadores pre- 
sentan una menor propensión a realizar deporte, quizás porque tienen diferente valoración sobre la importancia de mantenerse en forma.

El lugar de residencia también influye en la participación deportiva. La probabilidad de realizar deporte activo y pasivo es mayor entre los habitantes de la capital, Pamplona, que concentra más de un tercio de la población de la región. Este efecto puede ser debido a que la oferta de instalaciones y de eventos deportivos es generalmente mayor en grandes ciudades, o a diferencias en las preferencias en la distribución del tiempo de ocio de la población en las zonas urbanas.

\section{Tabla 2}

Probit bivariante de participación individual en actividades deportivas y de asistencia a espectáculos deportivos con carácter regular

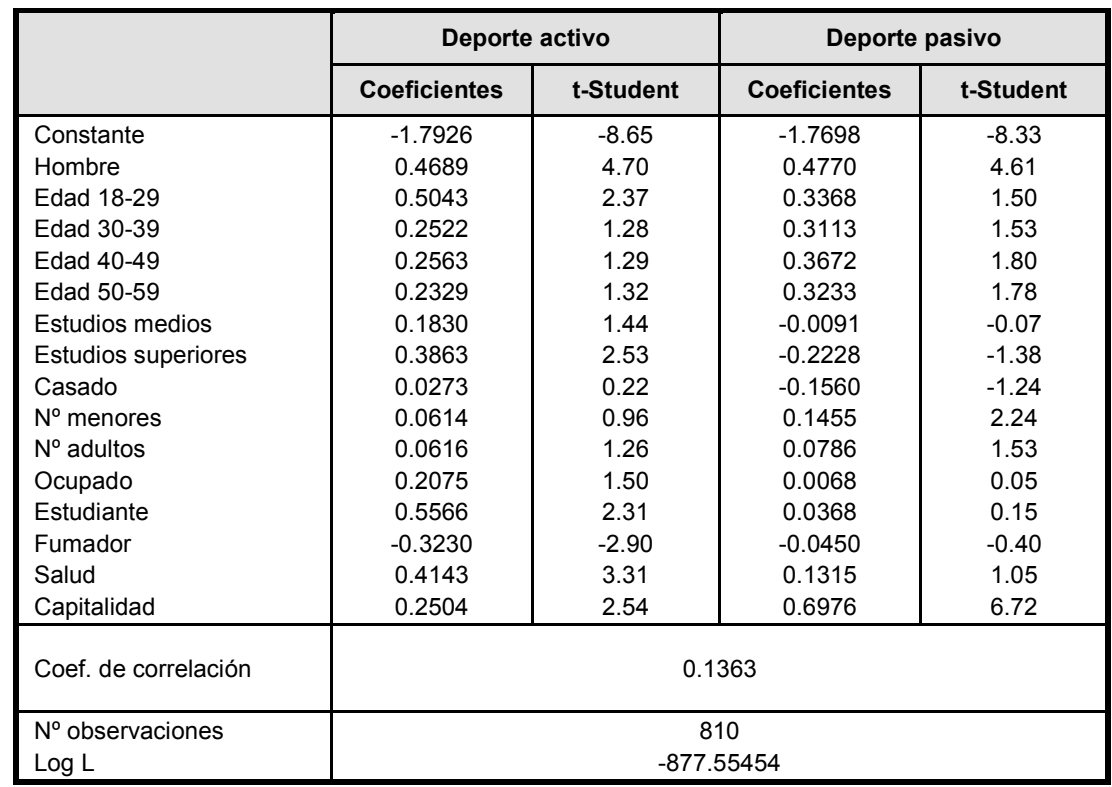

Fuente: Elaboración propia.

La situación laboral, que se incluyó fundamentalmente como una proxy de la renta, no afecta significativamente ni a la probabilidad de practicar deporte ni a la de asistir a espectáculos deportivos.

En cuanto a la composición familiar, el estado civil y el número de adultos en el hogar no afectan significativamente a la participación en deporte activo y pasivo. En cambio, el número de menores aumenta la probabilidad de asistir a eventos deportivos. 
Finalmente, el coeficiente de correlación entre los términos aleatorios de ambas ecuaciones de participación es positivo. Para comprobar su significatividad, se ha realizado un test de razón de verosimilitudes, en el que la hipótesis nula es que el coeficiente es igual a cero y el valor del test nos permite rechazar dicha hipótesis con un nivel de significatividad del 5\%. Este resultado implica que existen variables inobservadas que afectan de forma directa y simultánea a las decisiones de participación en deporte activo y pasivo.

La segunda especificación propuesta para el análisis de la participación individual en actividades deportivas de carácter activo y pasivo es un Logit multinomial, en el que la variable explicada toma 4 posibles valores, tal como se ha explicado en la sección 3.1. Los resultados de la estimación se muestran en la Tabla 3, en la que se ha tomado como categoría base la alternativa de no practicar deporte ni asistir a eventos.

\section{Tabla 3}

Logit multinomial de participación individual en actividades deportivas y de asistencia a espectáculos deportivos con carácter regular

\begin{tabular}{|c|c|c|c|c|c|c|}
\hline & \multicolumn{2}{|c|}{$\begin{array}{l}\text { Asiste a eventos y no } \\
\text { practica deporte }\end{array}$} & \multicolumn{2}{|c|}{$\begin{array}{l}\text { No asiste a eventos y } \\
\text { practica deporte }\end{array}$} & \multicolumn{2}{|c|}{$\begin{array}{l}\text { Asiste a eventos y } \\
\text { practica deportes }\end{array}$} \\
\hline & Coeficientes & $\begin{array}{c}\mathrm{t}- \\
\text { Student }\end{array}$ & Coeficientes & $\begin{array}{c}\mathrm{t}- \\
\text { Student }\end{array}$ & Coeficientes & $\begin{array}{c}\mathrm{t}- \\
\text { Student }\end{array}$ \\
\hline Constante & -2.5965 & -6.12 & -2.7003 & -6.58 & -6.5845 & -8.47 \\
\hline Hombre & 0.7197 & 3.15 & 0.7384 & 3.78 & 1.5519 & 5.73 \\
\hline Edad 18-29 & 0.3851 & 0.79 & 0.7679 & 1.86 & 1.6694 & 2.66 \\
\hline Edad 30-39 & 0.2785 & 0.65 & 0.2885 & 0.75 & 1.3275 & 2.23 \\
\hline Edad 40-49 & 0.4094 & 0.96 & 0.2885 & 0.74 & 1.3962 & 2.33 \\
\hline Edad 50-59 & 0.5454 & 1.48 & 0.4093 & 1.19 & 0.9807 & 1.74 \\
\hline Estudios medios & 0.0600 & 0.22 & 0.3869 & 1.52 & 0.1851 & 0.53 \\
\hline Estudios superiores & -0.3005 & -0.80 & 0.7760 & 2.60 & 0.0996 & 0.25 \\
\hline Casado & -0.3521 & -1.33 & 0.0042 & 0.02 & -0.0118 & -0.03 \\
\hline $\mathrm{N}^{\circ}$ menores & 0.2810 & 1.92 & 0.1149 & 0.90 & 0.2992 & 1.93 \\
\hline $\mathrm{N}^{\circ}$ adultos & 0.0832 & 0.76 & 0.0636 & 0.65 & 0.3224 & 2.43 \\
\hline Ocupado & -0.1406 & -0.47 & 0.1962 & 0.73 & 0.5071 & 1.28 \\
\hline Estudiante & -0.6408 & -0.99 & 0.5282 & 1.12 & 1.0526 & 1.81 \\
\hline Fumador & 0.0000 & 0.00 & -0.5061 & -2.31 & -0.5511 & -1.97 \\
\hline Salud & 0.0720 & 0.29 & 0.5824 & 2.35 & 1.0298 & 2.70 \\
\hline Capitalidad & 1.1229 & 4.83 & 0.3028 & 1.56 & 1.5297 & 5.59 \\
\hline $\begin{array}{l}\mathrm{N}^{\circ} \text { observaciones } \\
\text { Log L }\end{array}$ & \multicolumn{6}{|c|}{$\begin{array}{r}810 \\
-873.275\end{array}$} \\
\hline
\end{tabular}

Nota: La categoría base es la opción de que el individuo no asiste a eventos ni practica deporte.

Fuente: Elaboración propia.

Centrándonos en los determinantes de la asistencia a eventos y de la práctica deportiva de forma regular -última columna de la Tabla 3-, observamos de nuevo que los hombres y los jóvenes son los que tienen una mayor probabilidad de elegir esta opción frente a la alternativa que se toma como base, que es no asistir a eventos ni practicar deporte. También es más probable que los estu- 
diantes y las personas que habitan en la capital practiquen deporte y acudan a espectáculos deportivos simultáneamente. El estado de salud ejerce una influencia positiva mientras que el hábito de fumar tiene el efecto contrario. Finalmente, el número de miembros del hogar (tanto menores de edad como adultos) tienen un efecto positivo sobre la probabilidad conjunta de participación en deporte activo y pasivo.

Para comparar las dos especificaciones propuestas hemos empleado el criterio de información de Akaike, dado que los modelos no están anidados. Según este criterio, el modelo más adecuado para analizar las decisiones de participación en deporte activo y pasivo es el Probit bivariante.

En conclusión, los resultados de ambas especificaciones en relación a los determinantes de la práctica deportiva y de la asistencia a espectáculos deportivos son relativamente similares. Además, las estimaciones del Probit bivariante nos permiten concluir que existe correlación positiva entre ambas decisiones, una vez que se controla el efecto de las variables socio-demográficas.

\subsection{Análisis empírico de los gastos individuales en actividades deportivas}

Tal como hicimos en la sección anterior para la participación, comenzamos nuestro análisis empírico analizando la distribución de la muestra en función del gasto en actividades deportivas de carácter activo y pasivo. Esta información está recogida en la Tabla 4. En ella se observa que es mucho más frecuente gastar en bienes y servicios relacionados con la práctica activa del deporte (un $85,8 \%$ de la muestra declara un gasto positivo) que en asistencia a espectáculos deportivos (el 39,8\% de la muestra). Conviene advertir que la asistencia a eventos deportivos incluye tanto eventos profesionales (con pago de una entrada) como la asistencia a eventos de carácter amateur. Por otra parte, la muestra se distribuye de manera uniforme entre los que gastan en ambos tipos de actividades o en ninguna, por un lado, y los que sólo declaran gastos en una de ellas, por otro lado.

\section{Tabla 4}

Distribución de la muestra en función del gasto en actividades deportivas activas y pasivas

\begin{tabular}{|l|c|c|c|}
\hline & $\begin{array}{c}\text { Gasto nulo en } \\
\text { eventos deportivos }\end{array}$ & $\begin{array}{c}\text { Gasto positivo en } \\
\text { eventos deportivos }\end{array}$ & No observaciones \\
\hline Gasto nulo en práctica deportiva & 86 & 14 & 100 \\
\hline Gasto positivo en práctica deportiva & 338 & 266 & 604 \\
\hline N $^{\circ}$ observaciones & 424 & 280 & 704 \\
\hline
\end{tabular}

Fuente: Elaboración propia. 
Para analizar las variables explicativas de las decisiones individuales de gasto en deporte activo y pasivo y la correlación entre ambas se ha estimado un Tobit bivariante, cuyos resultados se encuentran en la Tabla 5.

\section{Tabla 5}

Tobit bivariante de gasto individual en actividades deportivas y en asistencia a espectáculos deportivos

\begin{tabular}{|c|c|c|c|c|}
\hline & \multicolumn{2}{|c|}{ Gasto en deporte activo } & \multicolumn{2}{|c|}{ Gasto en deporte pasivo } \\
\hline & Coeficientes & t-Student & Coeficientes & t-Student \\
\hline Constante & -16.0625 & -2.76 & -61.1623 & -7.41 \\
\hline Hombre & 7.6891 & 2.45 & 18.3359 & 4.76 \\
\hline Edad 18-29 & 17.0710 & 2.56 & 9.2896 & 1.12 \\
\hline Edad 3039 & 15.6499 & 2.52 & 18.1808 & 2.39 \\
\hline Edad $40-49$ & 13.3025 & 2.17 & 18.6205 & 2.47 \\
\hline Edad 50-59 & 8.8162 & 1.60 & 16.6324 & 2.44 \\
\hline Estudios medios & 5.8861 & 1.46 & 6.0192 & 1.21 \\
\hline Estudios superiores & 19.4209 & 3.93 & 0.3953 & 0.06 \\
\hline Casado & 4.1286 & 1.11 & -4.6956 & -1.02 \\
\hline $\mathrm{N}^{\circ}$ menores & 2.0293 & 0.98 & -1.4371 & -0.58 \\
\hline $\mathrm{N}^{\circ}$ adultos & 2.4591 & 1.58 & 3.2444 & 1.74 \\
\hline Ocupado & 7.4668 & 1.73 & 1.6295 & 0.31 \\
\hline Estudiante & 13.9351 & 1.78 & 14.2199 & 1.57 \\
\hline Fumador & -0.4956 & -0.14 & 11.0988 & 2.73 \\
\hline Salud & 1.0428 & 0.27 & 10.0430 & 2.09 \\
\hline Capitalidad & 11.0280 & 3.54 & 9.9247 & 2.59 \\
\hline Coef. de correlación & \multicolumn{4}{|c|}{0.450} \\
\hline $\begin{array}{l}\mathrm{N}^{\circ} \text { observaciones } \\
\text { Log } \mathrm{L}\end{array}$ & \multicolumn{4}{|c|}{$\begin{array}{c}704 \\
-4784.7035\end{array}$} \\
\hline
\end{tabular}

Fuente: Elaboración propia.

Al igual que sucedía con la participación, los hombres gastan más en bienes y servicios relacionados con la práctica del deporte y con la asistencia a eventos deportivos, confirmando la evidencia empírica disponible. En relación a la edad, el gasto esperado en actividades deportivas activas es mayor cuanto más joven es el individuo, mientras que el gasto en asistencia a espectáculos deportivos es significativamente mayor en los tramos intermedios de edad (entre 30 y 59 años).

Por otra parte, un alto nivel educativo ejerce una influencia positiva sobre el gasto en deporte activo, lo que es consistente con los resultados obtenidos en España, Alemania y Países Bajos. Por el contrario, la educación no afecta al gasto destinado a espectáculos deportivos, señalando la falta de evidencia empírica concluyente al respecto.

Las características familiares no determinan los gastos en deporte activo y pasivo, salvo el número de adultos, que tiene una relación directa con el gasto en asistencia a eventos. Por otra parte, los estudiantes y los trabajadores gastan 
más en práctica deportiva. Los fumadores y las personas que declaran un buen estado de salud tienen un mayor gasto en asistencia a espectáculos deportivos. Finalmente, el lugar de residencia ha resultado también un factor determinante de los gastos dedicados a ambos tipos de actividades..

Por último, como ocurrió en el Probit bivariante expuesto en la sección anterior, una vez controlado el efecto de las variables explicativas, se observa una correlación positiva entre el gasto en asistencia y el gasto en práctica deportiva. Para contrastar la hipótesis de que el coeficiente de correlación es igual a cero, se ha empleado un test de razón de verosimilitudes y la conclusión es que este coeficiente es distinto de cero con un nivel de significatividad del 1\%. Además su valor es relativamente elevado, lo que corrobora nuestra hipótesis inicial de que existen factores que afectan simultáneamente a las dos categorías de gasto.

\section{RESUMEN Y CONCLUSIONES}

Este artículo tiene carácter empírico y su objetivo es analizar la relación existente entre las decisiones individuales de deporte activo y pasivo, tanto en el ámbito de la participación como del gasto. Para ello se emplea una base de datos realizada en Navarra en el año 2007 a una muestra de personas adultas con el objetivo fundamental de recabar información sobre hábitos deportivos.

La encuesta ofrece datos sobre práctica de actividades deportivas o ejercicio físico, gastos en bienes y servicios asociados a la práctica del deporte, asistencia a espectáculos deportivos de carácter profesional o amateur, y gastos relacionados con dicha asistencia. Además, también pregunta acerca de características personales y familiares.

En el análisis de la participación, se especifican y estiman un modelo Probit bivariante, para estudiar la posible correlación entre deporte activo y pasivo, y un Logit multinomial, que permite analizar conjuntamente los determinantes de la probabilidad de realizar deporte y de asistir a espectáculos deportivos con regularidad. En el ámbito del gasto, se estima un modelo Tobit bivariante de gastos en cada una de las dos actividades.

Los resultados obtenidos indican que los hombres y los jóvenes tienen mayor probabilidad de participar en deporte activo y pasivo, y tienden a gastar más. La práctica deportiva y el gasto asociado a esta actividad son mayores para las personas más educadas. Sin embargo, ni la probabilidad de asistir a eventos ni el gasto correspondiente varían con el nivel educativo. La composición familiar es relevante en algunos casos. El estado de salud ejerce una influencia positiva sobre la práctica deportiva y la asistencia a espectáculos deportivos, mientras que hábitos poco saludables, como ser fumador, reducen la probabilidad de realizar deporte y aumentan el gasto en asistencia a eventos deportivos. 
En relación al objetivo principal de nuestro artículo, tanto en la participación como en el gasto hemos detectado una correlación positiva entre el deporte activo y pasivo. Esto implica que hay factores no observados que aumentan la probabilidad de practicar deporte y de asistir a espectáculos simultáneamente, y lo mismo se puede decir respecto al gasto. La principal implicación de este resultado es que las políticas que fomenten la realización de actividades deportivas también pueden tener un efecto positivo sobre la asistencia a espectáculos deportivos, y viceversa.

De este modo, el presente trabajo se enmarca dentro de la evidencia empírica mostrada por otros trabajos en el Reino Unido (Dawson y Downward, 2011) y los países escandinavos (Thrane, 2001) y de la corriente de autores que justifican una relación entre ambas modalidades de práctica deportiva (Zilmann et al., 1979; Sloan, 1989). Por ello, en el marco del desarrollo de políticas públicas de promoción de la práctica deportiva, estrategias de patrocinio público de eventos deportivos, tanto de carácter profesional o amateur, pudieran justificarse por el impacto positivo sobre la práctica deportiva.

Entre las posibles extensiones de este trabajo, se encuentra la de realizar un análisis similar con una base de datos nacional, para comprobar si se confirman los resultados obtenidos. Una segunda extensión interesante consistiría en enlazar participación y gasto a través de modelos de decisión secuencial, y analizar conjuntamente el deporte pasivo y activo, permitiendo correlación entre todas las variables. Asimismo, si se dispusiese de una base de datos amplia, se podría tratar de manera separada la asistencia a eventos deportivos de carácter profesional y de carácter amateur, y desagregar también la práctica deportiva según distintas modalidades deportivas.

\section{REFERENCIAS BIBLIOGRÁFICAS}

ALONSO, J.; RUESGA, S.; SÁEZ; F. y VICENS, J. (1991): "Impacto económico del deporte en España". Revista de Investigaciones y Documentación sobre las Ciencias de la Educación Física y el Deporte, 18, pp. 22-35.

BARBER, N. y HAVITZ, M.E. (2001): "Canadian participation rates in ten sport and fitness activities". Journal of Sport Management, 15(1), pp. 51-76.

BENITTEZ ROCHER, J. y LACOMBA ARIAS, B. (2012): "Los avances en la valoración económica del deporte en Europa". Estudios de Economía Aplicada, 30(2), pp. 1-18.

BOSCH, J., CAPEL, L., COUGOULE; F., FERRARI, G., SIERA; D., y SOLANAS; S. (2012): "El peso económico del deporte en Cataluña en 2006", Estudios de Economía Aplicada, 30(2), pp. 1-28. 
BREUER, C.; HALLMANN, K.; WICKER, P. y FEILER, S. (2010): "Socio-economic patterns of sport demand and ageing". European Revue Aging Physical Activity, 7, pp. 61-70.

BREUER, C. y WICKER, P. (2008): "Demographic and economic factors influencing inclusion in the German sport system. A microanalysis of the years 1985 to 2005". European Journal for Sport and Society, 5(1), pp. 33-42.

BURNETT, J.; MENON, A. y SMART, D.T. (1993): "Sports marketing: a new ball game with new rules". Journal of Advertising Research, 33(5), pp.21-35.

BYON, K.; ZHANG, J. y CONNAUGHTON, D. (2010): "Dimensions of general market demand associated with professional team sports: Development of a scale". Sport Management Review, 13(2), pp. 142-157.

CAMERON, A.C. y TRIBEDI, P.K. (2009): Microeconometrics using Stata. Ed, Stata Press.

CANNON, T. y FORD, J. (2002): "Relationship of demographic and trip characteristics to visitor spending: an analysis of sports travel visitors across time". Tourism Economics, 8(3), pp. 263-271.

CRAMER, J.S. (1991), The logit model: An introduction for economists. Ed, E. Arnold.

DAVIES, L. (2002): “Consumer's expenditure on sport in the United Kingdom: Increased spending or underestimation?" Managing Leisure, 7, pp. 83-102.

DAWSON, P. y DOWNWARD, P.M. (2011): "Participation, Spectatorship and Media Coverage in Sport: Some initial insights". En Andreff, W. (ed.): Contemporary Issues in Sports Economics, Participation and Professional Sports (pp. 15-42). Cheltenham: Edward Elgar.

DOWNWARD, P. (2007): "Exploring the economic choice to participate in sport: Results from the 2002 General Household Survey". International Review of Applied Economics, 21, pp. 633-653.

DOWNWARD, P.; DAWSON, A. y DEJONGHE, T. (2009): Sports economics: theory, evidence and policy. Oxford: Elsevier.

DOWNWARD, P.; LERA-LÓPEZ, F. y RASCIUTE, S. (2011): "The Zero-inflated ordered probit approach to modelling sports participation". Economic Modelling, 28, pp. 2469-2477.

DOWNWARD, P.; LERA-LÓPEZ, F. y RASCIUTE, S. (2012): "The economic analysis of sports participation". En Robinson, Chelladurai, Bodet y Downward (eds), Routledge Handbook of Sport Management (pp.331-353). London: Routledge.

DOWNWARD, P. y RASCIUTE, S. (2010): "The relative demands for sports and leisure in England”. European Sport Management Quarterly, 10(2), pp. 189214.

EBERTH, B. y SMITH, M. (2010): "Modelling the participation decision and duration of sporting activity in Scotland". Economic Modelling, 27, pp. 822-834. 
EUROPEAN FOOTBALL STATISTICS (2009): Historical attendances. http://www.european-football-statistics.co.uk. (Último acceso: Diciembre 2011).

FALTER, J. y PÉRIGNON, C. (2000): "Demand for football and intramatch winning probability: an essay on the glorious uncertainty of sports". Applied Economics, 32, pp. 1757-1765.

FERNÁNDEZ, V. y PRIETO, J. (2001): Are live sports substitute of cultural consumption? Some evidence for the Spanish case. Porto: Vugata.

FRIDBERG, T. (2010): "Sport and exercise in Denmark, Scandinavia and Europe". Sport in Society, 13(4), pp. 583-592.

GARCÍA, J. y RODRÍGUEZ, P. (2002): "The determinants of football match attendance revisited: Empirical evidence from the Spanish football league". Journal of Sports Economics, 3(1), pp. 18-38.

GARCÍA, J. y RODRÍGUEZ, P. (2009): "Sports attendance: A survey of the literature 1973-2007". Revista di Diritto Ed Economia Dello Sport, 5(2), pp. 111-151.

GARCÍA, J.; LERA-LÓPEZ, F. y SUÁREZ, M.J. (2011): "Estimation of a structural model of the determinants of the time spent on physical activity and sport: Evidence for Spain". Journal of Sports Economics, 12(5), pp. 18-38.

GARCÍA, M. y LLOPIS, R. (2011): Ideal democrático y bienestar personal. Encuesta sobre los hábitos deportivos en España 2010. Madrid: CIS y Consejo Superior de Deportes.

HOVEMANN, G. y WICKER, P. (2009): "Determinants of sport participation in the European Union". European Journal for Sport and Society, 6(1), pp. 51-59.

HUMPHREYS B. y RUSESKI, J.E. (2007): "Participation in physical activity and government spending on parks and recreation". Contemporary Economic Policy, 25, pp. 538-552.

HUMPHREYS B., y RUSESKI, J.E. (2010): The economic choice of participation and time spent in physical activity and sport in Canada. Working Paper No 2010-14, Department of Economics, University of Alberta.

KOKOLAKAKIS, T.; LERA-LÓPEZ, F.y PANAGOULEAS, T. (2012): "Analysis of the determinants of sports participation in Spain and England". Applied Economics, 44(21), pp. 2785-2798.

LAMB, L.L.; ASTURIAS, L.P.; ROBERTS, K. y BRODIE, D.A. (1992): "Sports participation -how much does it cost?". Leisure Studies, 11(1), pp. 19-29.

LECHNER, M. (2009): "Long-run labour market and health effects of individual sports activities". Journal of Health Economics, 28, pp. 839-854.

LERA-LÓPEZ, F. y RAPUN, M. (2005): "Sports participation versus consumer expenditure on sport: Different determinants and strategies in sports management". European Sport Management Quarterly, 5(2), pp. 167-186.

LERA-LÓPEZ, F. y RAPÚN, M. (2007): "The demand for sport: Sport consumption and participation models". Journal of Sport Management, 21, pp. 103-122. 
LERA-LÓPEZ, F.; RAPÚN, M. y AGUIRRE, J. (2008): Análisis y evaluación económica de la participación deportiva en España. Madrid: Consejo Superior de Deportes.

LERA-LÓPEZ, F.; RAPÚN, M. y SUÁREZ, M.J. (2011): "Determinants of individual consumption on sports attendance in Spain". International Journal of Sport Finance, 6(3), pp. 204-221.

LINES, G. (2007): "The impact of media sport events on the active participation of young people and some implications for pedagogy". Sport, Education and Society, 12, pp. 349-366.

LOYLAND, K. y RINGSTAD, V. (2009): "On the price and income sensitivity-of the demand for sports: Has Linder's disease become more serious?". Journal of Sports Economics, 10(6), pp. 601-616.

MONTGOMERY, S. y ROBINSON, M. (2010): "Empirical evidence of the effects of marriage on male and female attendance at sports and arts". Social Science Quarterly, 9(1), pp. 99-116.

MUÑIZ, C.; RODRÍGUEZ, P. y SUÁREZ, M.J. (2011): "The allocation of time to sports and cultural activities: An analysis of individual decisions". International Journal of Sport Finance, 6(3), pp. 245-264.

PAWLOWSKI, T. y BREUER, C. (2011): "The demand for sports and recreational services: Empirical evidence from Germany". European Sport Management Quarterly, 11(1), pp. 5-34.

PAWLOWSKI, T. y BREUER, C. (2012): "Expenditure elasticities of the demand for leisure services". Applied Economics, 44(26), pp. 3461-3477.

SCHEERDER J. y VOS, S. (2011): "Social stratification in adults' sports participation from a time-trend perspective. Results from a 40-year household study". European Journal for Sport and Society, 8(1-2), pp. 31-44.

SCHEERDER, J.; VANREUSEL, B., y TAKS, M. (2005): "Stratification patterns of active sport involvement among adults: Social change and persistence". International Review for the Sociology of Sport, 40 pp. 139-162.

SCHEERDER, J.; VOS, S. y TAKS, M. (2011): "Expenditure on sport apparel: Creating consumer profiles through interval regression modelling". European Sport Management Quarterly, 11(3), pp. 251-274.

SERRANO, J.A. (1999): "Prácticas y audiencias deportivas". Revista Española de Investigaciones Sociológicas, 87, pp. 101-134.

SHAMIR, B. y RUSKIN, H. (1984): "Sports participation vs. sport spectatorship: Two modes of leisure behavior". Journal of Leisure Research, 16, pp. 9-21.

SIGÜENZA, W. y MARIEL, P. (2012): "Determinantes de la pertenencia a entidades deportivas". Estudios de Economía Aplicada, 30(2), pp. 1-22.

SLOAN, L.R. (1989): "The motives of sport fans". En Goldstein J.H. (ed). Sports, games and play: Social and psychology viewpoints (pp. 175-240). Hillsdale, $\mathrm{NJ}$ : Erlbaum Associates. 
STONE, G.P. (1971): "American sports: Play and display". En Dunning, E. (ed.), The sociology of sport. (pp. 47-65). London: Frank Cass-Co.

TAKS, M.; RENSON, R. y VANREUSEL, B. (1995): "Social stratification in sport: a matter of money or taste?". European Journal for Sport Management, 2(1), pp. 4-14.

TAKS, M.; RENSON, R. y VANREUSEL, B. (1999): "Consumer expenses in sport: a marketing tool for sports". European Journal for Sport Management, 6(1), pp. 4-18.

TAKS, M. y SCHEERDER, J. (2006): "Youth sports participation styles and market segmentation profiles: Evidence and application". European Sport Management Quarterly, 6(2), pp. 85-121.

THRANE, C. (2001): "Sport spectatorship in Scandinavia. A class phenomenon?". International Review for the Sociology of Sport, 36, pp. 149-163.

VAN TUYCKOM, C.; SCHEERDER, J. y BRACKE, P. (2010): "Gender and age inequalities in regular sports participation: A cross-national study of 25 European countries". Journal of Sports Sciences, 28(10), pp. 1077-1084.

WHITE P. y WILSON, B. (1999): "Distinctions in the stands: An investigation of Bourdieu's habitus, socioeconomic status and sport spectatorship in Canada". International Review for the Sociology of Sport, 34, pp. 245-264.

WICKER, P.; BREUER, C., y PAWLOWSKI, T. (2009): "Promoting sport for all age-specific target groups. The impact of sport infrastructure". European Sport Management Quarterly, 9, pp. 103-118.

WICKER, P.; BREUER, C. y PAWLOWSKI, T. (2010): "Are sports club members big spenders? Findings form sport specific analyses in Germany". Sport Management Quarterly, 13, pp. 214-224.

WILSON, T.C. (2002): "The paradox of social class and sports involvement: The roles of cultural and economic capital". International Review for the Sociology of Sport, 37(1), pp. 5-16.

WINFREE, J.; McCLUSKEY, J.; MITTELHAMMER; R. y FORT, R. (2004): “Location and attendance in major league baseball". Applied Economics, 36819), pp. 2117-2124.

ZHANG, J.J.; LAM E.C.; BENNETT, G. y CONNAUGHTON, D.P. (2003): "Confirmatory factor analysis of the spectator decision making inventory". Measurement in Physical Education and Exercise Science, 7(2), pp. 57-70.

ZHANG, J.J.; SMITH, D.W.; PEASE D.G. y JAMBOR, E.A. (1997): "Negative influence of market competitors on the attendance of professional sport games: the case of a minor league hockey team". Sport Marketing Quarterly, 6(3), pp. 31-40.

ZILMAN, D.; BRYANT, H. y SAPOLSKY, B.S. (1979): "The enjoyment of watching sport contests". En Goldstein J. (ed.), Sports, games and play (pp. 297-335). New Jersey: L. Elbraum Assoc. 


\section{APÉNDICE}

Tabla A.1.

Estadísticos descriptivos

\begin{tabular}{|c|c|c|c|c|}
\hline \multirow[t]{2}{*}{ Variable } & \multicolumn{2}{|c|}{$\begin{array}{c}\text { Muestra empleada en } \\
\text { el Probit Bivariante y } \\
\text { en el Logit } \\
\text { multinomial } \\
\left(\mathrm{N}^{\circ} \text { obs. }=810\right)\end{array}$} & \multicolumn{2}{|c|}{$\begin{array}{l}\text { Muestra empleada en } \\
\text { el Tobit Bivariante } \\
\left(N^{\circ} \text { obs. }=704\right)\end{array}$} \\
\hline & Media & $\begin{array}{l}\text { Desviación } \\
\text { típica }\end{array}$ & Media & $\begin{array}{l}\text { Desviación } \\
\text { típica }\end{array}$ \\
\hline Deporte activo $=1$ si practica deporte al menor una vez al mes ${ }^{3}$, & 0.348 & 0.477 & --- & --- \\
\hline $\begin{array}{l}\text { Deporte pasivo = } 1 \text { si asiste a eventos deportivos al menos una } \\
\text { vez al mes. }\end{array}$ & 0.265 & 0.442 & --- & --- \\
\hline $\begin{array}{l}\text { Gasto en deporte activo = gasto mensual en cuotas deportivas, } \\
\text { uso instalaciones, clases, calzado y ropa, material deportivo, } \\
\text { desplazamientos, etc. }\end{array}$ & --- & --- & 29.825 & 38.153 \\
\hline $\begin{array}{l}\text { Gasto en deporte pasivo = gasto mensual en asistencia a eventos } \\
\text { deportivos, incluyendo cuotas socio, entradas, desplazamientos, } \\
\text { refrescos, comidas y productos de promoción adquiridos en el } \\
\text { evento. }\end{array}$ & --- & --- & 10.185 & 23.118 \\
\hline Hombre & 0.494 & 0.500 & 0.486 & 0.500 \\
\hline Edad 18-29 & 0.226 & 0.418 & 0.227 & 0.419 \\
\hline Edad 30-39 & 0.165 & 0.372 & 0.156 & 0.363 \\
\hline Edad 40-49 & 0.177 & 0.382 & 0.185 & 0.388 \\
\hline Edad 50-59 & 0.170 & 0.376 & 0.175 & 0.380 \\
\hline $\begin{array}{l}\text { Estudios medios = } 1 \text { si el individuo ha realizado estudios } \\
\text { secundarios o formación profesional }\end{array}$ & 0.446 & 0.497 & 0.452 & 0.498 \\
\hline $\begin{array}{l}\text { Estudios superiores = } 1 \text { si el individuo ha realizado estudios } \\
\text { universitarios }\end{array}$ & 0.198 & 0.398 & 0.196 & 0.397 \\
\hline Casado = 1 si está casado o viviendo con pareja & 0.619 & 0.486 & 0.615 & 0.487 \\
\hline Número de menores en el hogar & 0.514 & 0.832 & 0.497 & 0.825 \\
\hline Número de adultos (mayores de 18 años) en el hogar & 2.626 & 1.077 & 2.597 & 1.072 \\
\hline $\begin{array}{l}\text { Ocupado = } 1 \text { si la persona trabaja actualmente, bien sea por } \\
\text { cuenta propia o ajena }\end{array}$ & 0.547 & 0.498 & 0.555 & 0.497 \\
\hline Estudiante $=1$ si la persona está estudiando actualmente & 0.081 & 0.274 & 0.072 & 0.259 \\
\hline Fumador = 1 si la persona fuma al menos dos cigarrillos al día & 0.294 & 0.456 & 0.295 & 0.457 \\
\hline Salud = 1 si el estado subjetivo de salud es bueno o muy bueno & 0.756 & 0.430 & 0.759 & 0.428 \\
\hline $\begin{array}{l}\text { Capitalidad = } 1 \text { si la persona reside en la capital de la región } \\
\text { (Pamplona) o comarca }\end{array}$ & 0.514 & 0.500 & 0.523 & 0.500 \\
\hline
\end{tabular}

Fuente: Elaboración propia.

${ }^{3}$ Las prácticas deportivas más usuales son la natación recreativa, el fútbol, la gimnasia de mantenimiento y el ciclismo recreativo. E1 61\% del gasto total está asociado a la práctica activa del deporte, mientras el 39\% restante está asociado a la asistencia a eventos deportivos. 
